This item is the archived peer-reviewed author-version of:

\title{
Hair ethyl glucuronide and serum carbohydrate deficient transferrin for the assessment of relapse in alcohol-dependent patients
}

\section{Reference:}

Crunelle Cleo, Verbeek Jef, Dom Geert, Covaci Adrian, Michielsen Peter, Cappelle Delphine, van Nuijs Alexander, Neels Hugo, et al..- Hair ethyl glucuronide and serum carbohydrate deficient transferrin for the assessment of relapse in alcohol-dependent patients

Clinical biochemistry - ISSN 0009-9120 - (2016), p. 1-6

Full text (Publishers DOI): http://dx.doi.org/doi:10.1016/j.clinbiochem.2015.11.023 


\section{Accepted Manuscript}

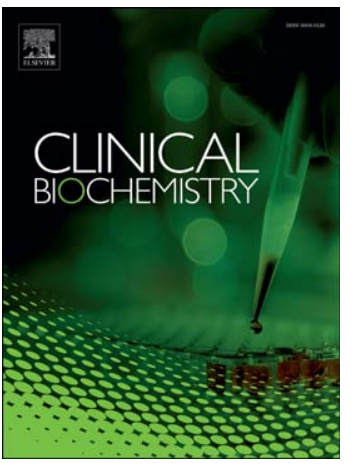

Hair Ethyl glucuronide and serum carbohydrate deficient transferrin for the assessment of relapse in alcohol-dependent patients

Cleo L. Crunelle, Jef Verbeek, Geert Dom, Adrian Covaci, Michel Yegles, Peter Michielsen, Mireille De Doncker, Frederik Nevens, Delphine Cappelle, Alexander L.N. van Nuijs, Hugo Neels

PII:

DOI:

S0009-9120(16)00051-5

Reference: doi: 10.1016/j.clinbiochem.2015.11.023

To appear in: $\quad$ Clinical Biochemistry

Received date: $\quad 7$ August 2015

Revised date: 6 October 2015

Accepted date: 18 November 2015

Please cite this article as: Crunelle Cleo L., Verbeek Jef, Dom Geert, Covaci Adrian, Yegles Michel, Michielsen Peter, De Doncker Mireille, Nevens Frederik, Cappelle Delphine, van Nuijs Alexander L.N., Neels Hugo, Hair Ethyl glucuronide and serum carbohydrate deficient transferrin for the assessment of relapse in alcohol-dependent patients, Clinical Biochemistry (2016), doi: 10.1016/j.clinbiochem.2015.11.023

This is a PDF file of an unedited manuscript that has been accepted for publication. As a service to our customers we are providing this early version of the manuscript. The manuscript will undergo copyediting, typesetting, and review of the resulting proof before it is published in its final form. Please note that during the production process errors may be discovered which could affect the content, and all legal disclaimers that apply to the journal pertain. 
Hair ethyl glucuronide and serum carbohydrate deficient transferrin for the assessment of relapse in alcohol-dependent patients

Running title: hair EtG and \%CDT to assess relapse

Cleo L. Crunelle ${ }^{1}(*)$, Jef Verbeek ${ }^{2}(*)$, Geert Dom ${ }^{3}$, Adrian Covaci ${ }^{1}$, Michel Yegles ${ }^{4}$, Peter Michielsen ${ }^{5}$, Mireille De Doncker ${ }^{6}$, Frederik Nevens ${ }^{2}$, Delphine Cappelle ${ }^{1}$, Alexander L. N. van Nuijs ${ }^{1}$, Hugo Neels ${ }^{1,6}$

(*) Authors contributed equally

${ }^{1}$ Toxicological Center, Department of Pharmaceutical Sciences, University of Antwerp, Antwerp, Belgium

${ }^{2}$ Department of Hepatology, University Hospital KU Leuven, Leuven, Belgium

${ }^{3}$ Collaborative Antwerp Psychiatric Research Institute, University of Antwerp, Antwerp, Belgium

${ }^{4}$ Laboratoire National de Santé, Service de Toxicologie, Dudelange, Luxembourg

${ }^{5}$ Department of Gastroenterology and Hepatology, University Hospital Antwerp, Antwerp, Belgium

${ }^{6}$ Toxicology and TDM Laboratory, ZNA Stuivenberg Hospital, Antwerp, Belgium

\section{Corresponding author:}

Cleo L. Crunelle

Toxicological Center, University of Antwerp,

Campus Drie Eiken, Universiteitsplein 1-S5.555, 2610 Antwerp, Belgium.

Email: cleo.crunelle@gmail.com / Phone: +32 (0)3 2652767

Word count: 3748

Tables / Figures: 3/2

Supplemental information: 0 


\section{Abstract}

Objectives: Ethyl glucuronide in hair (hEtG) and serum carbohydrate deficient transferrin $(\% \mathrm{CDT})$ are valuable markers for alcohol abuse, but their diagnostic accuracy to monitor abstinence and relapse is unclear. Here, we investigate to what extent repeated measurements of $\mathrm{hEtG}$ and \%CDT can be used to monitor relapse in alcohol-dependent patients during abstinence treatment.

Design and methods: HEtG and \%CDT were measured in individuals starting treatment for alcohol dependence both at treatment entry and 3 months later. Alcohol consumption and relapse episodes were recorded using the Time Line Follow Back and by alcohol breath and urine tests, and correlated with hEtG and \%CDT measurements.

Results: Fifteen patients completed the study, of which nine had one or more relapses. Hair EtG and serum \%CDT identified whether a relapse occurred in $78 \%$ and $57 \%$ of cases, respectively. Only hEtG correlated with the amount of alcohol consumed before treatment entry (Pearson $\mathrm{r}=0.92 ; p<0.001$ ). The specificity of $\% \mathrm{CDT}$ to assess abstinence during treatment was $100 \%$. HEtG had a specificity of only $17 \%$; however, in all patients who remained abstinent, hEtG decreased with $>85 \%$ from initial values. Mean $\mathrm{hEtG}$, but not $\% \mathrm{CDT}$, differed significantly between patients who relapsed and patients who remained abstinent $(p=0.034)$.

Conclusions: HEtG was more sensitive than serum \%CDT to assess relapse in alcoholdependent patients and was positively correlated with the amounts of alcohol consumed. In contrast, serum \%CDT was more specific for assessing abstinence. We highlight the benefit of repeated measurements of hEtG and serum \%CDT for monitoring abstinence during treatment.

Keywords: ethyl glucuronide; carbohydrate deficient transferrin; hair; alcohol; relapse; abstinence assessment 


\section{Introduction}

Monitoring abstinence in alcohol-dependent patients is highly relevant in both legal and medical settings. The detection of alcohol abstinence and relapse can have a major impact on legal decisions for individuals convicted for alcohol related offenses or individuals in high risk work settings. Also, it influences healthcare decisions such as the eligibility for liver transplantation in case of end stage alcoholic cirrhosis or the intensification of psychiatric support [1-2].

Direct measurement of ethanol in blood or breath remains the golden standard to detect recent alcohol use. However, the short half-life of ethanol of several hours decreases the sensitivity and thus utility of these methods to detect alcohol relapses in the prior weeks. The limited diagnostic value of indirect markers such as liver tests, reflecting tissue damage, fueled the search for additional biomarkers. Carbohydrate deficient transferrin $(\% \mathrm{CDT})$ is an iron transporter protein that can be detected in serum to assess excessive alcohol use [3-4]. Improvements in the analytical techniques to quantify serum \%CDT levels have increased its sensitivity and specificity [5-6]. Serum \%CDT has a half-life of circa 15 days and thus gives an indication of the alcohol use in the last 2 to 4 weeks [7]. Ethyl glucuronide (EtG) is a phase II metabolite of alcohol that accumulates in hair [8]. Studies have shown that EtG in scalp hair (hEtG) is a highly sensitive and specific marker for the detection of chronic moderate and excessive alcohol consumption [8-9]. HEtG concentrations in the proximal $3 \mathrm{~cm}$ hair length correlate well with the consumed amount of alcohol in the last 3 months in alcohol-dependent individuals [10]. HEtG thus has a broader detection window compared with ethanol blood levels and serum \%CDT. On the other hand, hEtG would have limited diagnostic value when assessing alcohol use of the past week compared to \%CDT, because EtG-containing hair needs to first grow out of the hair follicle to the visible hair part to be detectable upon analysis. To address both time windows (recent use and chronic use), it has been suggested 
that $\mathrm{hEtG}$ and \%CDT biomarkers may be combined for a more complete picture of both previous and current alcohol use [11].

The kinetic behavior of serum \%CDT and hEtG in alcohol-dependent patients after alcohol withdrawal has been underreported. A large multicenter study showed that \%CDT decreased significantly from initial levels between 1 and 4 weeks following abstinence, and that relapse during the course of the study correlated with a $30 \%$ increase in $\%$ CDT levels in the first week following the relapse [12]. For hEtG, a study in 15 alcohol-dependent patients showed that hEtG declined only moderately after the start of abstinence, probably due to the presence of non-growing hair [8]. Earlier studies comparing \%CDT and hEtG investigated abstinence assessment after chronic alcohol consumption [13-14], thereby lacking repeated measurements (i.e., within one individual). These studies were performed in the context of drunk driving where alcohol use in the prior months could be underreported [13-14], whereas the latter study [13] reports hEtG results with a cut-off of $30 \mathrm{pg} / \mathrm{mg}$ hair which is not representative for abstinence assessment (Society of Hair Testing (SoHT) guideline on hEtG [15]). Information on the duration of abstinence required for a negative test outcome is of major importance for the interpretation of a single test result in order to detect alcohol relapse or confirm sustained abstinence [16]. So far, few studies focused on the assessment of alcohol relapse based on multiple measurements [16]. We hypothesize that the combined measurement of serum \%CDT and hEtG will be an improved decision tool especially for the assessment of alcohol relapse over a broad time window. Therefore, we investigated whether $\mathrm{hEtG}$ and/or \%CDT can be used as an objective tool for the detection of relapse in alcoholdependent patients.

\section{Material and Methods}

\subsection{Participants}


Alcohol-dependent individuals starting an in-patient treatment for alcohol dependence were recruited from a Belgian addiction treatment center (Broeders Alexianen, Boechout, Belgium). Patients were required to have hair length $>3 \mathrm{~cm}$ and be $>18$ years old. Patients were excluded from study participation if they entered an alcohol detoxification program in the prior 3 months or if they had an alcohol-free period of more than 7 consecutive days in the 3 months prior to study inclusion. Patients with liver and kidney diseases were also excluded (assessed by serum transaminases, bilirubin and creatinine laboratory test values $>3 \mathrm{x}$ reference interval). The work has been carried out in accordance with The Code of Ethics of the World Medical Association (Declaration of Helsinki). The study was approved by the Ethics Committee of the Antwerp University Hospital (registration number: B300201215330) and all included patients signed informed consent.

\subsection{Questionnaires and sample collection}

Alcohol consumption was recorded using the Alcohol Timeline Follow Back Interview (TLFB; [17]), a widely used and validated tool to assess daily retrospective alcohol consumption and relapse [18]. Alcohol consumption was recorded at baseline (i.e., at treatment entry) to assess retrospective alcohol consumption in the three months prior to entry, and was re-assessed following three months treatment to assess relapse. Urine alcohol and alcohol breath tests were performed by the treatment center sporadically as part of the clinical routine and the information was used to verify the relapses documented by patients on the TLFB at the end of the study. Age, gender and body mass index (BMI) were recorded at baseline. Hair characteristics (length, color, daily washing and cosmetic treatments) were assessed by interview at baseline and three months later.

At baseline and 3 months later, a strand of hair (approximately $50 \mathrm{mg}$ ) was collected from the vertex posterior as close as possible to the scalp for hEtG measurements, in 
accordance with the Consensus on Hair Testing guidelines provided by the SoHT (see further). In parallel, blood from the arm vein was collected in a serum tube $(4 \mathrm{cc})$ for $\% \mathrm{CDT}$ measurements (see further).

\subsection{Hair EtG measurements}

Collected hair strands were stored in aluminum foil at room-temperature until analysis. The proximal $3 \mathrm{~cm}$ hair segment was used for hEtG measurement, in order to obtain data on alcohol consumption of the prior three months. Hairs were washed with water and acetone to remove external contamination, and pulverized to powder using a ball mill (Retsch MM400). Of the pulverized sample, $30 \mathrm{mg}$ was carefully weighted and used for further analysis using gas chromatography-mass spectrometry (GC/MS) in negative chemical ionization mode following solid-phase extraction on Oasis MAX cartridges and derivatization with pentafluoropropionic anhydride (PFPA), as reported earlier [19]. Our protocol for the measurement of EtG in hair is presented in Table 1. The limit of detection (LOD) was 0.7 $\mathrm{pg} / \mathrm{mg}$ and the lower limit of quantification (LLOQ) was $2.1 \mathrm{pg} / \mathrm{mg}$. The method validation was reported earlier [20]. According to the SoHT guidelines, a $\mathrm{hEtG}$ result $\geq 7 \mathrm{pg} / \mathrm{mg}$ is considered a positive result, i.e. indicates regular alcohol consumption. A hEtG result $\geq 30$ $\mathrm{pg} / \mathrm{mg}$ proposes chronic excessive alcohol consumption of $>60 \mathrm{~g}$ alcohol per day over several months [15]. External quality control was assessed through participation to two international inter-laboratory comparison schemes organized by the SoHT (www.soht.org) and by Arvecon (www.arvecon.de).

$$
===\text { Insert Table } 1 \text { about here }==
$$




\subsection{Serum CDT measurements}

Collected serum tubes were centrifuged at $5000 \mathrm{rpm}$ for $10 \mathrm{~min}$ and the serum was stored at $20^{\circ} \mathrm{C}$ until analysis. \%CDT was analyzed using capillary electrophoresis on the Capillarys 2 (Sebia). A cut-off of $1.6 \%$ ( $\%$ of di-sialo-transferrin compared to total transferrin) was used as a positive test result [6].

\subsection{Statistics}

Statistical analyses were performed using SPSS IBM software version 20, and all data were checked for normality using Shapiro-Wilk tests. Patient characteristics, alcohol consumption, $\mathrm{hEtG}$ concentrations and \%CDT measurements between relapsing and abstinent patients were analyzed using independent sample T-tests (for parametric data), Mann-Whitney U tests (for non-parametric data) or Chi-square tests (for frequencies). Linear regression analyses were used to analyze and describe partial correlations between hEtG, \%CDT, and alcohol consumption. For hEtG, we investigated alcohol consumption (on the TLFB) of the prior three months for comparison with hEtG concentrations. For \%CDT, due to its shorter half-life $[7,21]$, correlations were investigated with alcohol consumption over the prior 1 month before \%CDT measurement. Finally, sensitivity (i.e., true positive rate), specificity (i.e., true negative rate), negative predictive value (NPV) and positive predictive value (PPV) were calculated. Data are described as mean \pm standard deviation (SD) or as median \pm interquartile range (IQR) where specified. A $p$-value $<0.05$ was considered statistically significant.

Sub-analyses were performed to additionally compare hEtG concentrations between patients who remained abstinent, patients who relapsed on $<10$ drinking days and on $>10$ drinking days in the past three months. In parallel, \%CDT measurements were compared between patients who had no drinking days in the past month, had $<10$ drinking days and had 
$>10$ drinking days in the past month. An ANOVA test was used to compare between-group differences, and student T-tests were used to assess post-hoc between-group differences.

\section{Results}

\subsection{Participants and hair characteristics}

Forty-two patients were included, of which 16 completed the study. Eleven patients withdrew informed consent, 6 patients left the treatment center and became untraceable, and 9 patients did not show up to the final study appointment. One patient claimed abstinence, while having three positive alcohol breath tests (all after the weekend during sporadic testing on Mondays at the treatment center) and was excluded from analyses. As a result, we present data of 15 patients who completed the study.

Patients remained in in-patient treatment for a mean of $67 \pm 49$ days, and 5 patients remained in in-patient treatment for the entire study protocol. Patients were $46 \pm 10$ years old, had a mean BMI of $24.8 \pm 3.0$, and were predominantly males (67\% males). Patients had blond $(\mathrm{N}=5)$, brown $(\mathrm{N}=7)$, or white $(\mathrm{N}=3)$ hairs. Three patients had colored hair, and none had bleached, permed or straightened their hairs in the prior 3 months.

\subsection{Baseline hEtG and serum CDT for the assessment of alcohol consumption}

At treatment entry, participants consumed a mean of $15603 \pm 6564 \mathrm{~g}$ alcohol over the total prior three months period (range $75-318 \mathrm{~g}$ /day; normally distributed: Shapiro-Wilk $p=$ 0.317). Hair EtG concentration ranged between 41.7 and $639.4 \mathrm{pg} / \mathrm{mg}(144.8 \pm 146.4 \mathrm{pg} / \mathrm{mg}$; median $\pm \mathrm{IQR}$ ) and were thus all above the cut-off of $30 \mathrm{pg} / \mathrm{mg}$ used to detect chronic excessive alcohol consumption. In contrast, serum \%CDT measurements were positive for only 6 participants (range $1.8-4.9 \%$ ), but negative for the 9 other participants (range 0.4 1.6\%). No participants were heterozygote for transferrin. This resulted in sensitivity for hEtG 
of $100 \%$ compared to a sensitivity for $\%$ CDT of $40 \%$ to detect chronic alcohol consumption prior to treatment entry.

There was a strong linear and positive correlation between the total amount of alcohol consumed in the three months prior to treatment entry and hEtG concentrations at treatment entry $(r=0.92 ; p<0.001)$. Serum \%CDT did not correlate with the amounts of alcohol consumed at baseline $(p=0.961)$.

\subsection{Hair EtG for the assessment of relapse}

Nine patients relapsed during the 3-month study period, consuming $800 \pm 7160 \mathrm{~g}$ alcohol (median $\pm \mathrm{IQR}$; range: $80-16000 \mathrm{~g}$ alcohol $/ 3$ months) on $9 \pm 50$ drinking days (median \pm IQR; range: 2 - 80 drinking days). Because of this large variation in amount of drinking days, sub-analyses with major and minor amount of drinking days are also described (see further). Relapse episodes were recorded on the TLFB and were corroborated by results of random alcohol tests at the treatment center. Six patients remained abstinent in the 3-month period. There were no statistically significant differences in age, gender distribution, BMI, and hair characteristics (color and cosmetic treatments) between the group of patients who relapsed and who remained abstinent (all $p>0.149$ ).

In 7 of 9 patients who relapsed during treatment, hEtG was positive with mean hEtG concentrations of $124.0 \pm 76.9 \mathrm{pg} / \mathrm{mg}$ (range $25.6-258.0 \mathrm{pg} / \mathrm{mg}$; Shapiro-Wilk $p=0.810$ ). Two patients who relapsed had hEtG results below the cut-off $7.0 \mathrm{pg} / \mathrm{mg}$, represented by concentrations of 6.4 and $2.8 \mathrm{pg} / \mathrm{mg}$. These two patients had minor relapses $(<10$ drinking days, see sub-analyses presented further). In patients who relapsed in the three months following treatment entry, we observed a positive linear correlation between the amount of alcohol consumed and $\mathrm{hEtG}$ concentrations $(\mathrm{r}=0.82 ; p=0.007)$. 
In patients who remained abstinent during treatment, $\mathrm{hEtG}$ concentrations decreased with $>85 \%$ from baseline $\mathrm{hEtG}$ measurement at treatment entry (mean $\mathrm{hEtG}$ change from baseline: $93.5 \% \pm 5.4 \%$; range 85.9 - $100.0 \%$ ). HEtG concentrations were <LLOQ (2.1 $\mathrm{pg} / \mathrm{mg}$ ) in only one participant. Four participants who remained abstinent had positive hEtG concentrations, however below the cut-off of $30 \mathrm{pg} / \mathrm{mg}$ (mean $13.1 \pm 2.4 \mathrm{pg} / \mathrm{mg}$; range $10.5-$ $15.2 \mathrm{pg} / \mathrm{mg}$; Shapiro-Wilk $p=0.628$ ), and one participant had a high hEtG concentration of $39.9 \mathrm{pg} / \mathrm{mg}$ hair while claiming abstinence. Together, mean hEtG concentrations were significantly higher in patients who relapsed compared to mean hEtG concentrations in patients who remained abstinent ( $p=0.034$; see Figure 1). Specificity, sensitivity, NPV and PPV are presented in Table 2.

$$
\begin{aligned}
& ===\text { Insert Figure } 1 \text { about here }== \\
& ===\text { Insert Table } 2 \text { about here }==
\end{aligned}
$$

\subsection{Serum \% CDT for the assessment of relapse}

Over the last month before \%CDT sample collection after 3 months, 7 patients relapsed and 8 remained abstinent. Amounts of alcohol consumed during relapse episodes in the prior month were $500 \pm 4140 \mathrm{~g}$ alcohol (median $\pm \mathrm{IQR}$; range: $10-6000 \mathrm{~g}$ alcohol $/$ month), with a median of $3 \pm 26$ drinking days (range: $1-30$ drinking days over the final month).

In patients who relapsed in the prior month, \%CDT values were positive in 4 of 7 patients (median \pm IQR: $1.90 \pm 2.25 \%$; range $1.90-4.90 \%$ ). \%CDT values in participants who remained abstinent in the prior month were all below cut-off (mean $0.68 \pm 0.32 \%$; range $0.20-1.30 \%$; Shapiro-Wilk $p=0.646)$. Sensitivity, specificity, PPV and NPV of \%CDT are 
presented in Table 2. In patients who relapsed and who remained abstinent in the prior month, $\%$ CDT did not significantly differ between groups $(p=0.094)$.

We observed a correlation between \%CDT measurements after 3 month treatment and the amounts of alcohol consumed during relapsing episodes in the prior month $(\mathrm{r}=0.71 ; p=$ $0.003)$.

\subsection{Sub-analyses between abstinent patients and patients with major and minor amount of drinking days during relapse on hEtG and serum \% CDT}

Because of a wide range of drinking days within patients who relapsed (from 2 to 80 over the past three months; and from 1 to 30 drinking days in the prior month), and taking into account the clinical significance of distinguishing full relapse from minor relapsing episodes, subanalyses were performed. A limit of $0,<10$ and $>10$ drinking days was used for analyses, to obtain groups of similar sample size for analyses.

HEtG concentrations were statistically different between groups of patients with $0,<$ 10 or $>10$ drinking days in the past 3 months (ANOVA $p=0.001$; see Table 2). Also, posthoc, hEtG concentrations were significantly different between patients with $<10$ drinking days and patients with $>10$ drinking days in the 3 -month treatment period $(p=0.022)$. No difference in mean hEtG was observed post-hoc between abstinent patients and patients who relapsed with $<10$ drinking days in these three months ( $p=0.175 ; \mathrm{NS})$.

For $\% \mathrm{CDT}$, we observed a difference between patients who remained abstinent and patients who relapsed with $<10$ and $>10$ drinking days (ANOVA $p=0.006$; see Table 3 ). However, post-hoc analyses showed that the differentiation could only be made between patients who remained abstinent and patients who relapsed with $>10$ drinking days in the prior month $(p=0.004)$, and not between patients who relapsed with $<10$ and $>10$ drinking 
days $(p=0.097$; NS) or between patients who remained abstinent and relapsed with $<10$ drinking days $(p=0.284$; NS).

$$
\begin{aligned}
& ==\text { Insert Table } 3 \text { about here }== \\
& ===\text { Insert Figure } 2 \text { about here }==
\end{aligned}
$$

\section{Discussion}

We show that hEtG is a sensitive marker to assess relapse in alcohol-dependent patients, correlating well with the ingested amount of alcohol in this period. Through repeated measurements, we provide insight in the kinetic behavior of increased $\mathrm{hEtG}$ levels in alcoholdependent patients after a period of abstinence.

All patients in the alcohol withdrawal program had hEtG levels $>30 \mathrm{pg} / \mathrm{mg}$ at inclusion, confirming the good sensitivity of hEtG for the detection of alcohol dependence [10]. HEtG levels decreased significantly in alcohol-dependent patients who remained abstinent for 3 months. But, remarkably, only one abstinent patient reached the proposed $\mathrm{hEtG}$ cut-off level for abstinence $(<7 \mathrm{pg} / \mathrm{mg})$. Thus, our data challenge the proposed $\mathrm{hEtG}$ cut-off level for abstinence in alcohol-dependent patients who remained abstinent for 3 months, but repeated measurements can show a significant reduction in hEtG levels that can be used to presume abstinence. This finding has major implications for legal or clinical decision-making. When applying this cut-off level in these patients, the low specificity will result in falsely accusing them of relapse and the subsequent refusal of working permits or even a life-saving procedure such as a liver transplantation. HEtG levels in patients with a recently installed abstinence might not be comparable with hEtG levels in abstinent patients who never had an alcohol use disorder or alcohol-dependent patients who remained abstinent for a longer time period. Most likely, this can be explained by the presence of hair in a non- 
growing cycle, lasting until 8 weeks, and that will represent $15 \%$ of the total amount of hair analyzed, as was presented in an earlier study [8]. These hairs in catagen or telogen phases will lead to $\mathrm{hEtG}$ values remaining positive for a longer time in the $0-3 \mathrm{~cm}$ hair segment in case of former heavy drinking. Our results indicate the need for further studies with repeated measurements on different time points after the start of abstinence and the simultaneous analysis of hEtG levels in different hair segments, as performed earlier [8]. This might lead to a more reliable use of $\mathrm{hEtG}$ cut-off levels to differentiate relapse from abstinence in patients with recent alcohol abuse. Moreover, the decrease of hEtG levels in all alcohol-dependent patients who remained abstinent suggest that this change itself might serve as the superior biomarker and might overcome inter-individual differences complicating the determination of cut-off levels. In this regard, an important point to take into consideration is the assessment of relapse, which was performed here using repeated personal interviews, and sporadic breathalyzer and urine alcohol tests, but not by urinary EtG. It is therefore still possible that, among the presumed abstainers, some may have had a covert relapse.

As for other biomarkers of alcohol consumption, several factors may influence the interpretation of EtG measurements, especially in a complicated matrix as hair. Many possible confounders have been investigated or are currently under investigation, which is of importance when interpreting hEtG concentrations that oppose self-report. For hEtG, it has been shown that the incorporation is not influenced by pigmentation [22], and neither by gender [10]. However, if the hairs have been bleached, colored, repeatedly styled or permed, the chance for a false negative result increases [23-24]. The use of hair lotions containing EtG has been described [25] and may lead to false positive results, but ethanol-containing products were found not to increase hEtG concentrations [26]. It is therefore important to record information on e.g., cosmetic hair treatments and lotions when obtaining a hair sample. However, compared to the measurement of $\% \mathrm{CDT}$, $\mathrm{hEtG}$ is a direct parameter, and its 
presence can be detected long before liver damage occurs.

This study confirms that \%CDT is a marker of recent alcohol relapse/abuse, given the higher sensitivity in patients who relapsed in the month prior to testing and the positive correlation with the consumed amount of alcohol in this period. The detection of minor relapse remains problematic. Two of the four patients with minor relapses in the prior month, defined as $<10$ drinking days, were not detected by hEtG or \%CDT. In addition, hEtG levels were not significantly different between abstinent patients and patients who relapsed with < 10 drinking days in the prior three months. Concerning the interpretation of $\% \mathrm{CDT}$ values, we should be aware of the \%CDT-nonresponders (i.e., the group of people who drink alcohol regularly without having increased \%CDT levels), as in these individuals even normal \%CDT values cannot exclude alcohol abuse.

In order to reduce the rate of false positive results, a \%CDT cut-off of $2.3 \%$ (instead of $1.6 \%$ ) is sometimes used, with values between $1.6 \%$ and $2.3 \%$ being reported as a 'grey zone' that needs retesting. When applying this higher cut-off to our patient sample, the sensitivity for $\% \mathrm{CDT}$ to detect chronic alcohol consumption prior to treatment entry was only minimally reduced from 40 to $33 \%$, i.e., only one participant had \%CDT in the grey zone, whereas 5 of 6 patients with $\%$ CDT values $>1.6 \%$ also had values $>2.3 \%$.

This study included a small number of participants, and our results need to be confirmed in a larger cohort of alcohol-dependent patients. The high drop-out rate (only 16 of 42 patients finalized the study) reflects the problematic compliance to the study protocol of this specific population, especially when repeated measurements for abstinence assessment are required. Another limitation is that the study observation period has been covered in full by $\mathrm{hEtG}$, but not by $\% \mathrm{CDT}$ (i.e., only the final month was addressed by $\% \mathrm{CDT}$ ). Analyzing more blood samples for \%CDT during the investigation period would have increased the comparability of the two data sets. Finally, advanced liver disease can impact the accuracy of 
\%CDT: cirrhosis can lead to an incomplete separation of disialotransferrin and trisialotransferrin, namely di- and trisialotransferrin-bridging. In our study, the patients had no history of liver disease and we found no patients with di- and trisialotransferrin-bridging.

\section{Conclusions}

Our study highlights the benefit of repeated measurements of hEtG and serum \%CDT for monitoring abstinence in alcohol-dependent patients during abstinence treatment. Caution is warranted when interpreting a single measurement of $\mathrm{hEtG}$ concentration in patients with recently installed abstinence and misclassifying them on the basis of current proposed cut-off levels. Therefore, repeated measurements of both biomarkers offer important future prospects.

\section{References}

[1] Dom, G., Marcin, W., Crunelle, C.L., Thon, N., Bobes, J., Preuss, U., Addolorato, G., Seitz, H., Wurst, F., 2015. Assessing and treating alcohol relapse risk in liver transplantation candidates: towards a European consensus. Alcohol Alcohol 50, 164172.

[2] Litten, R.Z., Bradley, A.M., Moss, H.B., 2010. Alcohol biomarkers in applied settings: recent advances and future research opportunities. Alcohol Clin. Exp. Res. 34, 955967.

[3] Delanghe, J.R. \& De Buyzere, M.L., 2009. Carbohydrate deficient transferrin and forensic medicine. Clin. Chim. Acta 406, 1-7.

[4] Stibler, H., Borg, S., Joustra, M., 1986. Micro anion exchange chromatography of carbohydrate-deficient transferrin in serum in relation to alcohol consumption (Swedish Patent 8400587-5). Alcohol Clin. Exp. Res. 10, 535-544. 
[5] Maenhout, T.M., Baten, G., De Buyzere, M.L., Delanghe, J.R., 2012. Carbohydrate deficient transferrin in a driver's license regranting program. Alcohol Alcohol 47, 253260.

[6] Schellenberg, F. \& Wielders, J.P., 2010. Evaluation of capillary electrophoresis assay for CDT on SEBIA's Capillarys System: intra and inter laboratory precision, reference interval and cut-off. Clin. Chim. Acta 411, 1888-1893.

[7] Stibler, H., Dahlgren, L., Borg, S., 1988. Carbohydrate-deficient transferrin (CDT) in serum in women with early alcohol addiction. Alcohol. 5, 393-398.

[8] Appenzeller, B.M., Agirman, R., Neuberg, P., Yegles, M., Wennig, R., 2007. Segmental determination of ethyl glucuronide in hair: a pilot study. Forensic Sci. Int. 173, 87-92.

[9] Crunelle, C.L., Yegles, M., van Nuijs, A.L., Covaci, A., De Doncker, M., Maudens, K.E., Sabbe, B., Dom, G., Lambert, W.E., Michielsen, P., Neels, H., 2014. Hair ethyl glucuronide levels as a marker for alcohol use and abuse: a review of the current state of the art. Drug Alcohol Depend. 134, 1-11.

[10] Crunelle, C.L., Cappelle, D., Covaci, A., van Nuijs, A.L., Maudens, K.E., Sabbe, B., Dom, G., Michielsen, P., Yegles, M., Neels, H., 2014. Hair ethyl glucuronide as a biomarker of alcohol consumption in alcohol-dependent patients: role of gender differences. Drug Alcohol Depend 141, 163-166.

[11] Neels, H., Yegles, M., Dom, G., Covaci, A., Crunelle, C.L., 2014. Combining serum carbohydrate-deficient transferrin and hair ethyl glucuronide to provide optimal information on alcohol use. Clin. Chem. 60, 1347-1348.

[12] Anton, R.F., Lieber, C., Tabakoff, B.; CDTect Study Group, 2002. Carbohydratedeficient transferrin and gamma-glutamyltransferase for the detection and monitoring of alcohol use: results from a multisite study. Alcohol Clin. Exp. Res. 26, 1215-1222. 
[13] Bianchi, V., Premaschi, S., Raspagni, A., Secco, S., Vidali, M., 2015. A comparison between serum carbohydrate-deficient transferrin and hair ethyl glucuronide in detecting chronic alcohol consumption in routine. Alc. Alc. 50, 266-270.

[14] Liniger, B., Nguyen, A., Friedrich-Koch, A., Yegles, M., 2010. Abstinence monitoring of suspected drinking drivers: ethyl glucuronide in hair versus CDT. Traffic Inj. Prev. 11, $123-126$.

[15] Cooper, G.A., Kronstrand, R., Kintz, P.; Society of Hair Testing, 2012. Society of Hair Testing guidelines for drug testing in hair. Forensic Sci. Int. 218, 20-24.

[16] Freeman, W.M. \& Vrana, K.E., 2010. Future prospects for biomarkers of alcohol consumption and alcohol-induced disorders. Alcohol Clin. Exp. Res. 34, 946-954.

[17] Sobell, L.C., Sobell, M.B., Leo, G.I., Cancilla, A., 1988. Reliability of a timeline method: assessing normal drinkers' reports of recent drinking and a comparative evaluation across several populations. Br J Addict. 83, 393-402.

[18] Carey, K.B., Carey, M.P., Maisto, S.A., Henson, J.M., 2004. Temporal stability of the timeline followback interview for alcohol and drug use with psychiatric outpatients. J Stud Alcohol. 65, 774-781.

[19] Kerekes, I., Yegles, M., Grimm, U., Wennig, R., 2009. Ethyl glucuronide determination: head hair versus non-head hair. Alcohol Alcohol. 44, 62-66.

[20] Kerekes, I. \& Yegles, M, 2013. Coloring, bleaching, and perming: influence on EtG content in hair, Ther. Drug Monit. 35, 527-529.

[21] Behrens, U.J., Worner, T.M., Lieber, C.S., 1988. Changes in carbohydrate-deficient transferrin levels after alcohol withdrawal. Alcohol Clin. Exp. Res. 12, 539-544.

[22] Appenzeller, B.M., Schuman, M., Yegles, M., Wennig, R., 2007. Ethyl glucuronide concentration in hair is not influenced by pigmentation. Alcohol Alcohol. 42, 326-237. 
[23] Crunelle, C.L., Yegles, M., De Doncker, M., Dom, G., Cappelle, D., Maudens, K.E., van Nuijs, A.L., Covaci, A., Neels, H., 2015. Influence of repeated permanent coloring and bleaching on ethyl glucuronide concentrations in hair from alcohol-dependent patients. Forensic Sci Int. 2015 247, 18-22.

[24] Ettlinger, J., Kirchen, L., Yegles, M., 2014. Influence of thermal hair straightening on ethyl glucuronide content in hair. Drug Test Anal. 6 S1, 74-77.

[25] Arndt, T., Schröfel, S., Stemmerich, K., 2013. Ethyl glucuronide identified in commercial hair tonics. Forensic Sci Int. 231, 195-198.

[26] Martins Ferreira, L., Binz, T., Yegles, M., 2012. The influence of ethanol containing cosmetics on ethyl glucuronide concentration in hair. Forensic Sci Int. 218: 123-125.

\section{Acknowledgements}

We kindly thank Mss. Karen Wouters and Mrs. Carine Van Mieghem with their help in interpreting the data.

\section{Role of funding source}

Nothing declared.

\section{Contributors}

H Neels and CL Crunelle designed the study. CL Crunelle and J Verbeek collected and analyzed the data and wrote the first draft. G Dom included the participating patients. A Covaci, M Yegles, M De Doncker and H Neels were involved in the interpretation of the data. F Nevens, A van Nuijs, D Cappelle, and P Michielsen commented on the draft and were involved in the preparation of the manuscript for submission. All authors reviewed and approved the final version. 


\section{Conflict of interest}

No conflict declared.

Table 1: Analytical protocol for the extraction and measurement of ethyl glucuronide in hair

Place $30 \mathrm{mg}$ of powdered hair sample in a vial and add

$20 \mu \mathrm{L} \mathrm{EtG-d5}(0.2 \mu \mathrm{g} / \mathrm{mL})$

$2 \mathrm{~mL}$ water

Close the vial, vortex for $15 \mathrm{~s}$, and ultrasonicate the sample for $2 \mathrm{~h}$

Centrifuge for $10 \mathrm{~min}$ at $2500 \mathrm{~g}$

Condition OASIS MAX cartridge with $2 \mathrm{~mL}$ methanol and $2 \mathrm{~mL}$ water

Place the aqueous phase of the sample on the cartridge and add

$1 \mathrm{~mL} \mathrm{5 \%} \mathrm{NH3} \mathrm{in} \mathrm{water}$

$2 \mathrm{~mL} \mathrm{MeOH}$

Dry the cartridge under vacuum

Add $2 \mathrm{~mL} \mathrm{2 \%}$ formic acid in methanol and dry the effluent using N2

For derivatization, add $100 \mu \mathrm{L}$ PFPA and vortex

Close immediately and heat to $60^{\circ} \mathrm{C}$ for $30 \mathrm{~min}$

Dry the sample with $\mathrm{N} 2$

Add $50 \mu \mathrm{L}$ ethyl acetate, vortex, and place in screw cap vial

Inject $1 \mu \mathrm{L}$ into the GC-MS system

Table 2. Overview of sensitivity, specificity, positive predictive value (PPV) and negative predictive value (NPV) of hair ethyl glucuronide (hEtG) and serum carbohydrate deficient transferrin $(\% \mathrm{CDT})$ to assess alcohol relapse in a population of 15 alcohol-dependent patients who started an alcohol detoxification program.

\begin{tabular}{|l|c|c|}
\hline & hEtG & \%CDT \\
\hline Sensitivity (relapse) & $78 \%$ & $57 \%$ \\
\hline Specificity (relapse) & $17 \%$ & $100 \%$ \\
\hline PPV (relapse) & $58 \%$ & $100 \%$ \\
\hline NPV (relapse) & $33 \%$ & $72 \%$ \\
\hline
\end{tabular}


Table 3. Overview of hair ethyl glucuronide $(\mathrm{hEtG})$ and \%CDT values in participants with no drinking days, $<10$ and $>10$ drinking days $\left({ }^{1}\right.$ in the past three months for EtG concentrations; in the past 30 days for $\%$ CDT measures). (*) Important for interpretation: one concentration was $<$ LLOQ and was included as value $0.0 \mathrm{pg} / \mathrm{mg}$ for calculation purposes.

\begin{tabular}{|l|c|c|c|c|}
\hline & $\begin{array}{c}\text { No drinking } \\
\text { days } 1\end{array}$ & $\begin{array}{c}<10 \text { drinking } \\
\text { days }\end{array}$ & $\begin{array}{c}>10 \text { drinking } \\
\text { days }\end{array}$ & $\begin{array}{c}p \text {-value } \\
(\text { ANOVA) }\end{array}$ \\
\hline EtG $(\mathrm{pg} / \mathrm{mg})$ & $13.7 \pm 14.2\left(^{*}\right)$ & $44.4 \pm 49.3$ & $163.8 \pm 73.7$ & 0.001 \\
& $($ sample $\mathrm{N}=6)$ & $($ sample $\mathrm{N}=5)$ & $($ sample $\mathrm{N}=4)$ & \\
\hline$\%$ CDT & $0.68 \pm 0.32$ & $1.00 \pm 0.70$ & $2.90 \pm 1.73$ & 0.006 \\
& $($ sample $\mathrm{N}=8)$ & $($ sample $\mathrm{N}=4)$ & $($ sample $\mathrm{N}=3)$ & \\
\hline
\end{tabular}

Figure 1: Mean difference in hair ethyl glucuronide (hEtG) concentrations between the group of patients who remained abstinent during treatment and the group of patients who relapsed during treatment. 


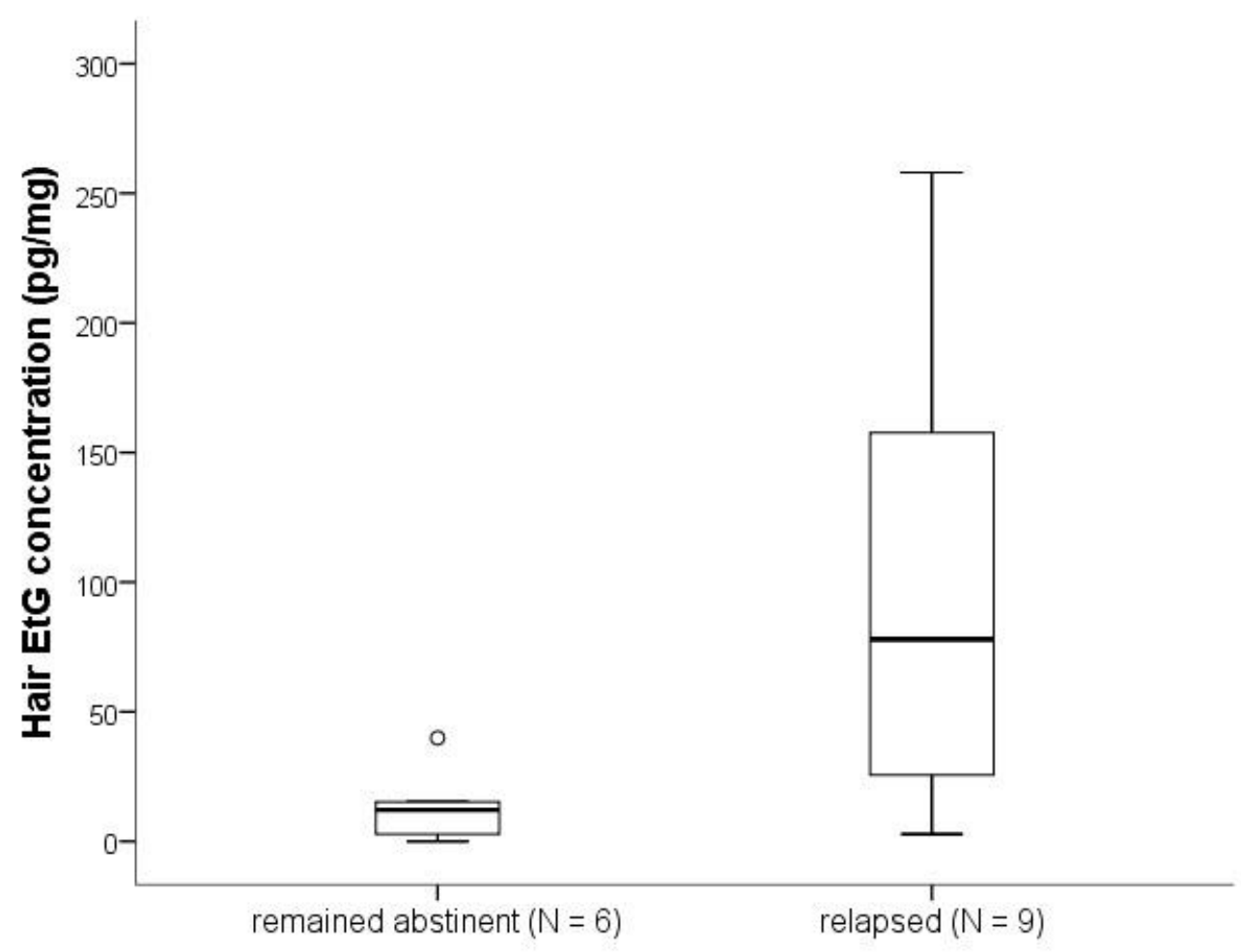

Figure 2: Sub-analyses showing the differences in hair ethyl glucuronide (hEtG) concentrations (A) and carbohydrate deficient transferrin (\%CDT; B) in participants that remained abstinent, and participants who had minor and major relapsing episodes in the prior 3 months (for $\mathrm{hEtG}$ ) or in the prior month (for \%CDT). 

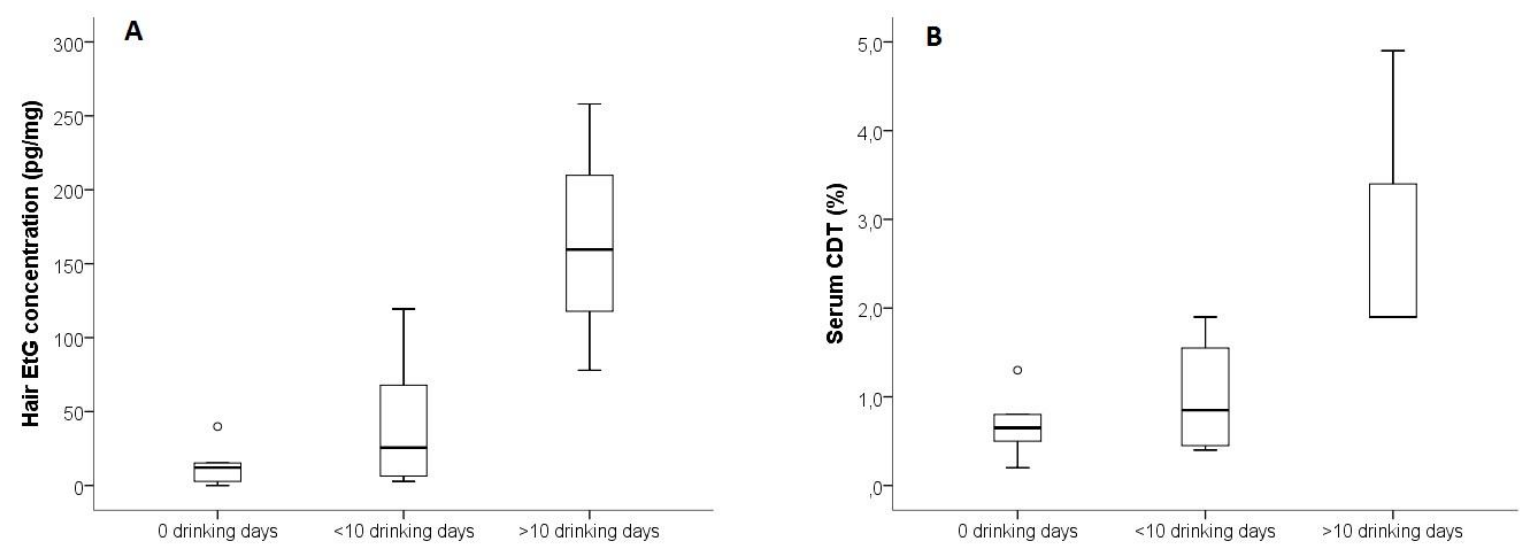

Highlights

- Hair testing is a valuable tool for the detection of alcohol use

- Hair ethyl glucuronide can be used to assess relapse in alcohol-dependent patients

- Serial measurements of hair EtG and serum \%CDT are useful for monitoring abstinence 